\title{
Geospatial analysis of invasion of the Asian tiger mosquito Aedes albopictus: competition with Aedes japonicus japonicus in its northern limit area in Japan
}

\author{
Naoko Nihei ${ }^{1,2 *}$, Osamu Komagata ${ }^{1 *}$, Kan-ichiro Mochizuki ${ }^{3}$, Mutsuo Kobayashi $^{1}$ \\ ${ }^{1}$ Department of Medical Entomology, National Institute of Infectious Diseases, Tokyo, Japan; ${ }^{2}$ Laboratory of \\ Parasitology, Department of Veterinary Medicine, Azabu University, Kanagawa, Japan; ${ }^{3}$ Pasco Research \\ Institute, Tokyo, Japan
}

\begin{abstract}
The mosquito Aedes albopictus, indigenous to Southeast Asia and nearby islands, has spread almost worldwide during recent decades. We confirm the invasion of this mosquito, first reported in Yamagata city in northeast Honshu, Japan in 2000. Previously, only Ae. japonicus japonicus had been collected in this place, but 2 years later, the population of Ae. albopictus had increased, so more than $80 \%$ of the total number of larval colonies there consisted of this species. In contrast to Yamagata's new residential area, now infested by Ae. albopictus, the original mosquito remains in the city but its habitats are generally closer to the surrounding mountains, where the normalized difference vegetation index is higher. The factors affecting the distribution of both species in Yamagata city were studied using geographical information systems (GIS) based on data derived from field surveys, aerial photographs, satellite images and digital maps. The range of Aedes mosquito habitats was estimated and visualised on polygon maps and no significant differences were noted when the polygon area was calculated by GIS software in comparison with the satellite images. Although Ae.j.japonicus was expected to be rapidly overrun by Ae. albopictus, this did not happen. Currently, both species coexist; not only in separate sites, but also simultaneously in various water bodies, where larvae from both species have frequently been seen. However, the competitive relationship between these two Aedes species within a warming environment is an issue that should be closely monitored.
\end{abstract}

Keywords: Aedes albopictus, Aedes japonicus japonicus, competition, normalized difference vegetation index, geographical information system, Japan.

\section{Introduction}

The mosquito, Aedes albopictus (Skuse, 1895) (Dipatera: Culicidae) represents one of the fastest spreading animal species in the world and has expanded from its native range of Southeast Asia and some islands in the western Pacific and Indian Ocean to at least 28 other countries around the globe during recent decades. This increase, blamed on the international trade in used tires that are often left unattended in the environment (Benedict et al., 2007), has been reported from Africa, the Middle East, Europe and the Americas (Hawley, 1989; Gratz, 2004; Benedict et al., 2007). Temperature is an important limiting variable restricting its establishment to areas with an annual mean temperature above $11^{\circ} \mathrm{C}$ (Kobayashi et al., 2002).

\footnotetext{
Corresponding author:

Naoko Nihei

Department of Medical Entomology

National Institute of Infectious Diseases

1-23-1 Toyama, Shinjuku-ku, Tokyo 162-8640, Japan

Tel. +81-3-5285-1111 ext. 2422; Fax +81-3-5285-1147

E-mail: niheigeo@nih.go.jp

* These authors contributed equally to the research
}

The current northern limit of Ae.albopictus in Japan ranges from the town of Ootsuchi in Iwate prefecture by the Pacific Ocean $\left(39^{\circ} 21^{\prime} 45^{\prime \prime} \mathrm{N}\right)$ to Morioka city in the Kitakami River basin in east of the Oou Mountains $\left(39^{\circ} 41^{\prime} 15^{\prime \prime} \mathrm{N}\right)$ and including the town of Happou in Akita prefecture by the Sea of Japan $\left(40^{\circ} 20^{\prime} 0^{\prime \prime} \mathrm{N}\right)$ in the Tohoku region (Fig. 1a) (Kobayashi et al., 2008; Sato et al., 2012). However, this limit has moved northward after the end of World War II due to temperature increases as shown by global climate models (GCMs), in particular by the K1coupled model for interdisciplinary research on climate (MIROC) developed by a team of Japanese research centres coordinated by the Center for Climate System Research, University of Tokyo (http://ccsr.aori.u-tokyo.ac.jp/ hasumi/miroc_ description.pdf). MIROC indicates that a further northern expansion into the Aomori prefecture can be expected by 2035, and into Hokkaido by 2100 (Kobayashi et al., 2008). Ae. albopictus is a laboratory-competent vector of seven alphaviruses, eight bunyaviruses and also three flaviviruses, i.e. Japanese encephalitis, West Nile fever and Yellow fever (Benedict et al., 2007). In Asia, this mosquito has been implicated as dengue vector in Southeast Asia, 
Seychelles, southern China and Japan (Hawley, 1989). Ae. albopictus is often viewed as insignificant with respect to dengue but, already in 1977, there was a major outbreak of dengue- 2 attributed to this vector on the island of Reunion affecting $30-35 \%$ of the inhabitants (Paupy et al., 2001). Between September 2001 and April 2002, 1,644 people on Hawaii were suspected of dengue infection with 122 confirmed cases at the same time as it was shown that the city of Nahiku in Hawaii, had a dense population of Ae.albopictus; interestingly without sighting of Ae. aegypti (Gratz, 2004). Another viral infection, in whose transmission Ae. albopictus may play a role, is chikungunya (CHIKV) fever; Reunion had one outbreak in 2005-2006 (Renault et al., 2007) and the European Centre for Disease Prevention and Control (ECDC) has reported another in northern Italy in 2007 (ECDC, 2009).

There is a debate regarding the competitive interaction between Ae.albopictus and Ae.aegypti, and it has been shown that male mosquitoes of Ae. albopictus transfer accessory gland proteins during mating that induce refractoriness to further mating in the female, which could explain the competitive displacement of resident Ae. aegpyti by Ae. albopictus (Tripet et al., 2011; Helinski et al., 2012). Ae.japonicus japonicus, another widespread and invasive species has become established in the United States of America (USA) and in parts of central Europe (Schaffner et al., 2003; Armistead et al., 2008; Werner et al., 2012). In Japan, Ae. albopictus and Ae.j.japonicus cohabit some environments, but the former tend to breed in urban environments and big city areas, while the latter is frequently collected in rural areas. Competitive displacement of Ae.j.japon$i c u s$ by Ae. albopictus has not been reported, but habitat segregation between the two species seems to have occurred. In this study, which took place in Yamagata city, which was not invaded by Ae. albopictus until 1998 (Kurihara et al., 1997; Kobayashi et al., 2008), we attempted to evaluate the effect of the invading Ae. albopictus mosquitoes on the population density of Ae.j. japonicus over a relatively long period of 12 years.

\section{Materials and methods}

In the year 2000, information was received of afternoon biting incidents some distance away from older urbanised areas in a new residential area developed on top of former paddy fields. A field survey was subsequently carried out and followed up in 2002, 2009 and 2010. In all, five surveys have been carried out including the first one in 1998.

\section{Study area}

Yamagata city is located in Tohoku district at latitude $38^{\circ} 15^{\prime} 9^{\prime \prime} \mathrm{N}$ and longitude $140^{\circ} 20^{\prime} 35^{\prime \prime} \mathrm{E}$; its elevation is $110-190 \mathrm{~m}$ above the mean sea level (MSL). The city lies south of a range of basins feeding the middle section of the Mogami River, which flows between the Oou Mountains (elevation $>1,300 \mathrm{~m}$ above MSL) that stretch in the north to south direction (Fig. 1a). The urbanised areas are located on the high, dry, alluvial fan terrain of the Umamigasaki (a)


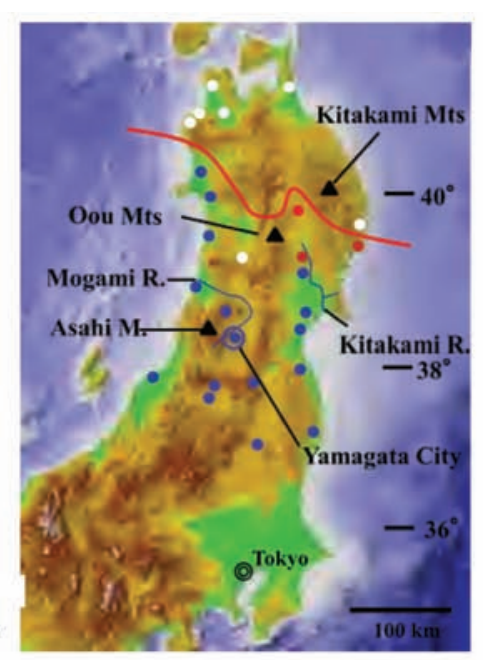

(b)

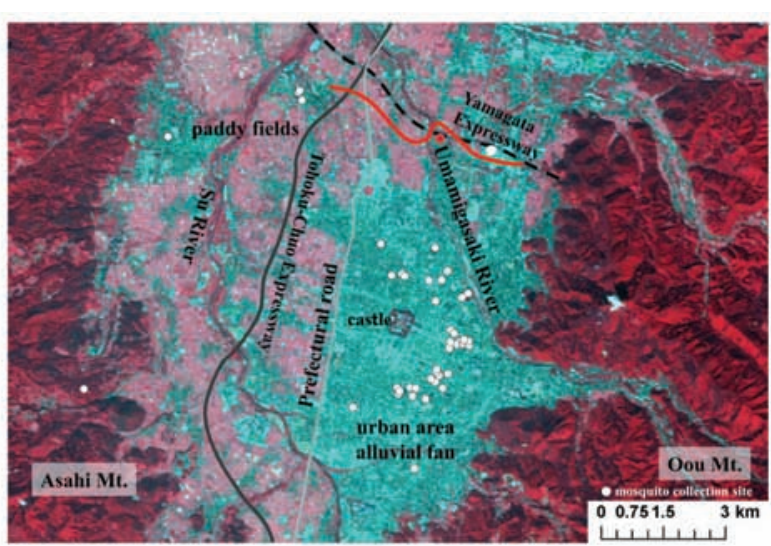

Fig. 1. Distribution of Ae. albopictus confirmed areas in the Tohoku region of Honshu in 2012 and the northern limit of Ae. albopictus. (b) Mosquito collection sites in Yamagata city (1998-2010) ALOS image of 19 September 2009 (false colour). 


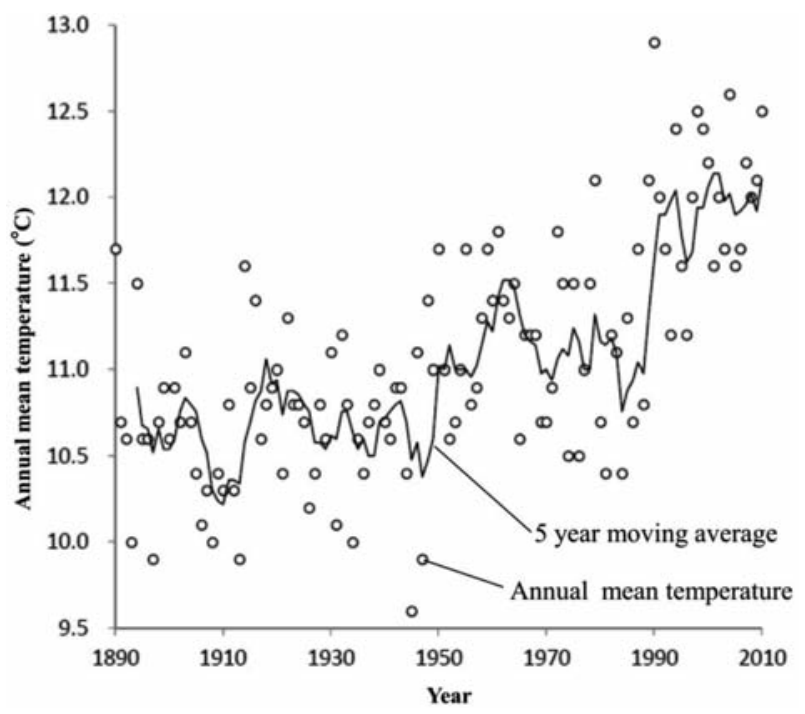

Fig. 2. Increases in annual mean temperature and its 5-year moving average. Data provided by the Yamagata local meteorological observatory.

River, a Mogami River tributary coming from the Oou Mountains (Fig. 1b). Rice paddy fields spread out across the alluvial lowlands of the Su River, another tributary, and across the edges of the alluvial fan area. National highway 51, which runs from north to south, marks the boundary of this area.

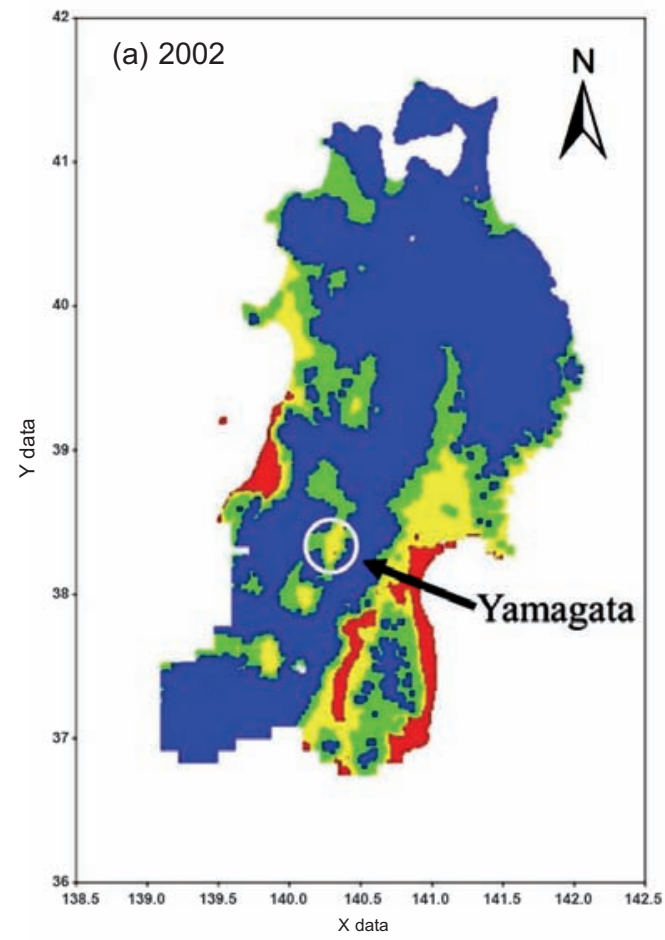

Temperatures

According to the Yamagata local meteorological observatory (YLMO), pre-war temperatures in the study area averaged $10^{\circ} \mathrm{C}$, while they averaged $11^{\circ} \mathrm{C}$ during the 1950 s- 1995 period. Since the annual fluctuations are large, 5-year moving averages provide a more even view (Fig. 2). The mean temperature repeatedly reached $12{ }^{\circ} \mathrm{C}$ in the 2000 s, indicating a continued rise. As the developmental zero point for Ae. albopictus is $11^{\circ} \mathrm{C}$, we created distribution maps for the areas with annual mean temperatures of $11^{\circ} \mathrm{C}$ for each year using the Tohoku region's $1 \mathrm{~km}$ mesh temperature data display/retrieval system (Tohoku Agricultural Research Center, NARO, 2009). The map for 2002 (Fig. 3a), featuring a low mean temperature, and the one for 2004 (Fig. 3b), featuring a high mean temperature, are shown here to demonstrate the significant differences.

The annual mean temperature is $11.2{ }^{\circ} \mathrm{C}(1961$ 1990), and the effective accumulative temperature for the threshold value of $11{ }^{\circ} \mathrm{C}$ is 1,500 degree-days (Kobayashi et al., 2002), with 169 days above $11^{\circ} \mathrm{C}$. These values approximate the boundary values for Ae. albopictus habitat according to Kobayashi et al. (2002).

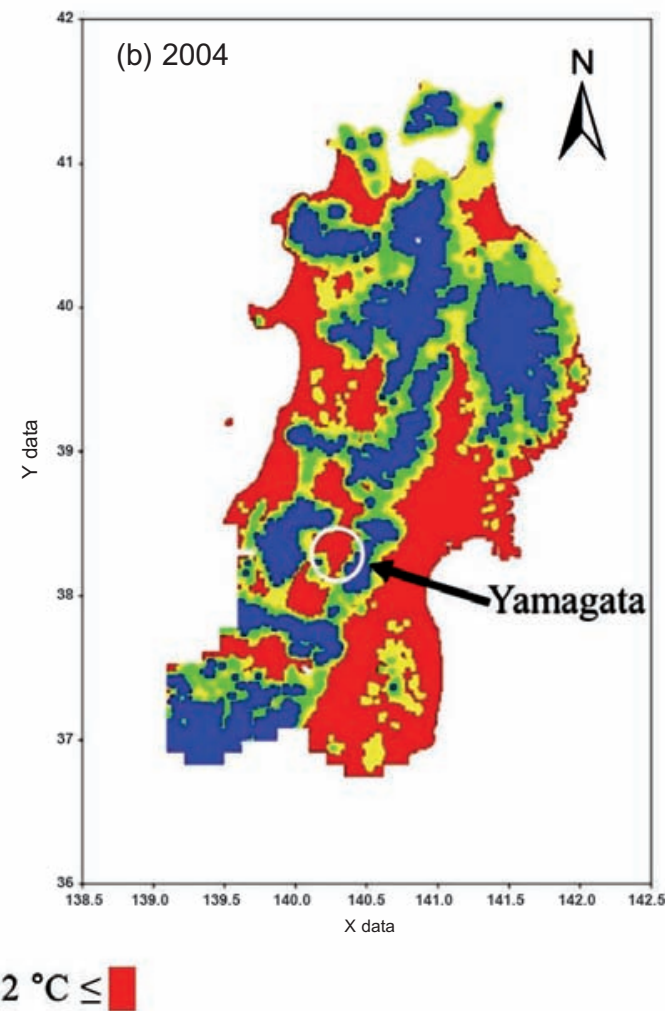

Fig. 3. Distribution maps for the areas with annual mean temperatures ranging from under $11{ }^{\circ} \mathrm{C}$ to $12{ }^{\circ} \mathrm{C}$ or above for 2002 and 2004. Figure based on the Tohoku Region's $1 \mathrm{~km}$ mesh temperature data display/retrieval system (Tohoku 599 Agricultural Research Center, NARO, 2009). 
Although the latitude of the study area is further south than the northern limit for Ae.albopictus, most of it consist of farming and mountain villages with urban areas isolated from neighbouring regions by mountains. Yamagata expressway opened for traffic in 1981 along the periphery of the urban areas of Yamagata city and the Yamagata bullet train connected the metropolitan area with the mass transit network in 1992.

\section{Specimen collection}

Larval and pupal specimens were collected from small, artificial water vessels, e.g. flower stands, incense holders and washbasins. Specimens were collected by pipettes including from water in abandoned tires. Each water body (including flower stands) was treated as one colony, and 5-10 specimens were taken from each one with 50 or more larvae and pupae. The specimens were placed in $100 \mathrm{ml}$ wide-mouth polypropylene containers half-filled with water and taken back to the laboratory. Water was changed as appropriate, and food was added to induce larval development; adult specimens were classified according to species and sex. Survey data were compiled with one temple treated as one collection site.

The 1998 survey area took place on 9 and 10 September and focused on the temple district in the mid-section of the alluvial fan covering urban areas across Yamagata city. Wider surveys were repeated on 2 and 30 September through 1 October 2000 and on 13 and 14 September 2002. In 2009, habitation surveys were resumed in order to investigate if changes had occurred during the 7 years after the first surveys, however now taking a larger number of samples from a wider area including areas east and north of the urbanised centre. Finally, this area was gone over again on 24 and 25 September 2010.

\section{Geographical support}

Digital maps, scale 1:2,500, were produced using a 2005 basic city planning map purchased from the Yamagata City Hall's Town Planning Promotion Department, Urban Policy Division. The digital national land information download services were provided by the National and Regional Planning Bureau of the Ministry of Land, Infrastructure and Transport. We also used aerial photographs with scales of approximately 1:10,000 (photo of 2007) and $1: 30,000$ (photo of 2003) that had been issued by the Geographical Information Authority of Japan.

Images from the Japan Aerospace Exploration Agency's advanced land-observing satellite (ALOS) dated 2 May, 17 September and 16 October 2008 along with images from 18 April, 18 June and 20 September 2009 were imported. Calculation of the normalized difference vegetation index (NDVI) values were done using band 3 (0.61-0.69 $\mu \mathrm{m})$ and band 4 (0.76$0.89 \mu \mathrm{m})$ and the visible and near-infrared radiometer JERS-1 AVNIR data from 10 October 1993, 18 June and 28 October 1995, 1 October 1997 and 26 May 1998 by band $2(0.63-0.69 \mu \mathrm{m})$ and band $3(0.76-$ $0.86 \mu \mathrm{m})$. Since the band wavelengths differ between the ALOS and JERS-1 images, the NDVI values of the survey areas were calculated for each scene and images were selected according to multivariate correlations.

Since the developmental zero point of Ae. albopictus in Shikoku, Japan is $11{ }^{\circ} \mathrm{C}$ (Udaka, 1959), the daily and annual mean temperatures, effective accumulative temperature with the threshold value of $11^{\circ} \mathrm{C}$ and the number of days per year above $11{ }^{\circ} \mathrm{C}$ were calculated. These climate data were retrieved by the $1 \mathrm{~km}$ mesh temperature data display/retrieval system of the Yamase climate research team of the National Agricultural and Research Center for Tohoku region. Only regions with annual mean temperatures above $11^{\circ} \mathrm{C}$ from 1961-1990 were mapped.

The ArcView 9.3.1 software (ESRI; Redlands, USA) was used for geographical information systems (GIS) analysis, and Imagine (Erdas Inc.; Norcross, USA) for analysis of the satellite images. The statistical analysis was supported by the JMP software (SAS Institute Inc.; Cary, USA).

\section{Mapping}

The larval survey results show the values calculated for each of the specimen collection sites, the type of vessel in which the larvae were found and their species and sex. Coordinates for the collection sites were determined using Google maps and the telephone numbers of temples or shrines and then transferred as point information on the digital maps created as described above.

In order to analyse larval habitat and habitat sites, excluding structures that were not assumed to provide habitats within a precinct yard and cemeteries, suitable areas were identified using the 1:2,500-scale city planning maps and aerial photographs, marking them onto the digital maps as polygons that were subsequently used as units for the environmental analysis. For quantitative analysis of the Ae. albopictus habitats, a NDVI map was created from the ALOS and JERS-1 images using ArcView. The habitat site polygons were then superimposed and the NDVI values compiled for them 
using the ArcView extension Spatial Analyst - Zonal Statistics. Of the NDVI maps, the ALOS image closest in date to the survey period and showing a high-vegetation index (19 September 2009) was chosen. Areas with NDVI values <-0.15 (no vegetation) and higher values (various degrees of vegetation) were identified in the aerial photographs. With respect to the NDVI results for the range of habitation polygons estimated from the Ae.albopictus and Ae.j. japonicus collection sites in 2009 , and the $100 \mathrm{~m}$ radius circles, the mean NDVI values were -0.053 and -0.104 , respectively, with average maximum values of 0.006 and 0.084 . Judging from these results, those with vegetation were classified into five ranges with NDVI values of -0.261 to $-0.104,-0.104$ to $-0.053,-0.053$ to $0.006,0.006$ to 0.084 and 0.084 to 0.359 , respectively.

Ae. albopictus and Ae.j.japonicus habitat ranges were estimated using local surveys and deciphering aerial photographs and maps. Significant differences depending on the collection site were noted. Because the unaided flight range of Ae. albopictus is thought to be within $100 \mathrm{~m}$ (Mori, 1979), habitats could be estimated by drawing circles of $100 \mathrm{~m}$ radius around the centroids of the estimated range of habitation polygons adjacent to the collection sites obtained via ArcGIS. The NDVI values of the estimated habitation polygons as well as of the $100 \mathrm{~m}$ radius circles were compared with ALOS images taken on 20 September 2009. Using the mean and maximum NDVI values of both spatial patterns as threshold values, we created distribution maps and then created an estimated habitation area map for both mosquito species in the areas surrounding Yamagata city.

\section{Results}

\section{Ae. albopictus infestation and establishment}

Miniature pie chart circles show the collection sites and the species-distribution of mosquito specimens for the surveys in the period 1998 to 2010 (Tables 1 and 3, Fig. 4). All specimens collected in 1998 (from 17 colonies at 10 sites) were Ae.j.japonicus (Fig. 4a), while the collection 2 years later harvested 11 sites producing 23 colonies, 12 of which $(52.2 \%)$ were Ae. albopictus ones and 11 Ae.j.japonicus ones $(47.8 \%)$ (Fig. 4b). In territorial terms, Ae. j. japonicus was prevalent in the temple districts to the east of the city, with Ae. albopictus mainly found in the temples of relatively new, urbanised areas. Coexistence of the two species was only confirmed in one area. When surveys were repeated in 2002 (harvesting from 37 colonies in 17 sites), 33 colonies $(86.8 \%)$ consisted of Ae. albopictus. In contrast, only five colonies $(13.2 \%)$ were pure Ae.j.japonicus ones (Fig. 4c) underlining the distinctly increased distribution of Ae. albopictus. The Ae.j.japonicus specimens came from colonies collected in coniferous groves of the Japanese shrines. Although coexistence of the two species was again confirmed in one area on the outskirts of the urbanised area, there was no case of both species coexisting in the same colony. Since it had been shown that Ae. albopictus was dominant already in 2002, it was inferred that Ae.albopictus was established in Yamagata city and survey activities were then halted.

When the situation was evaluated in 2009, the results showed that Ae.j.japonicus was exclusively confirmed in some areas east and north of the urbanised area and, out of 86 colonies in 21 areas, 49 $(57 \%)$ consisted only of Ae. albopictus and 24 $(27.9 \%)$ only of $A e . j . j a p o n i c u s$, while 13 colonies $(15.1 \%)$ consisted of both species in coexistence (Fig. $4 \mathrm{~d})$. When looking only at survey areas in the mid-section of the alluvial fan, many sites (11) consisted of habitats with both species coexisting, while four exclusive Ae.albopictus sites and three exclusive Ae. j. japonicus sites were confirmed. With a comparatively high number of areas in which both species coexisting, the state of affairs seems to be more complicated than an "either or" situation that was originally contemplated. Over the entire survey period, exclusive Ae.j.japonicus habitation was only confirmed in the forests of Shinto shrines along the Umamigasaki River to the east. In 2010, Aedes spp.

Table 1. Overview of the specimen collection suveys.

\begin{tabular}{lcccc}
\hline Year & Time point & Area & No. of colonies & No. of sites \\
\hline 1998 & $9-10$ September & Temple district & 17 & 10 \\
2000 & 2 and 30 September* & Temple district & 23 & 11 \\
2002 & $13-14$ September & Temple district & 37 & 17 \\
2009 & $18-20$ August & Urban area and environs & 86 & 21 \\
2010 & $24-25$ September & Urban area and environs & 126 & 33 \\
\hline
\end{tabular}

*Through 1 October in some places 

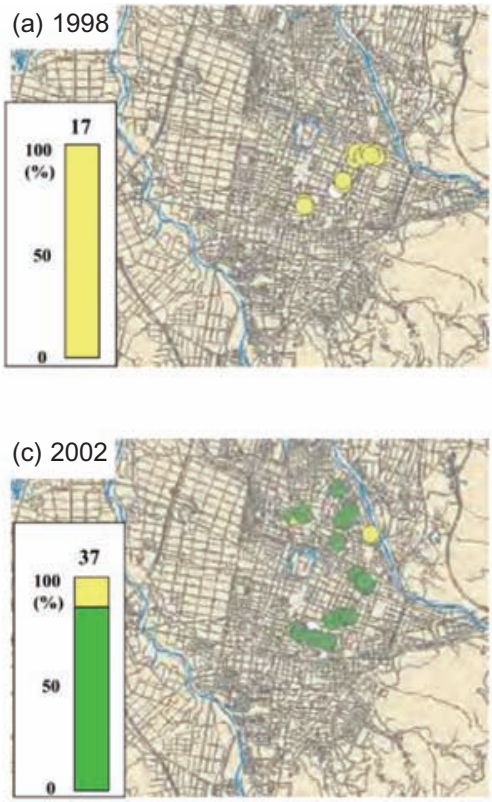
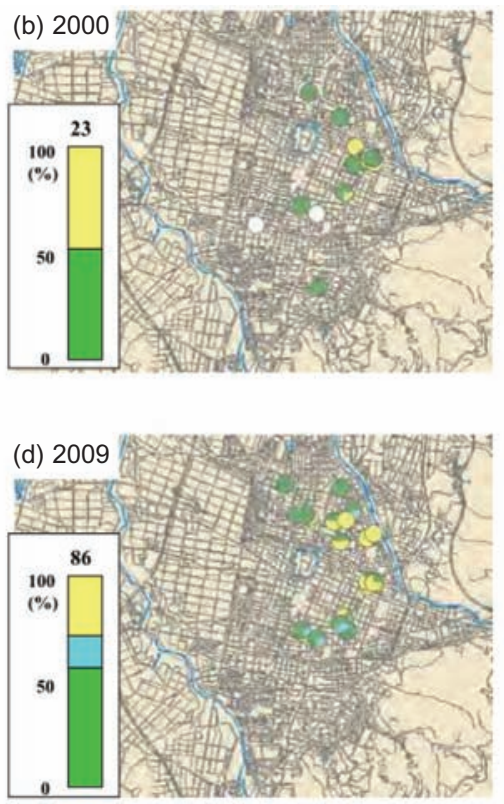

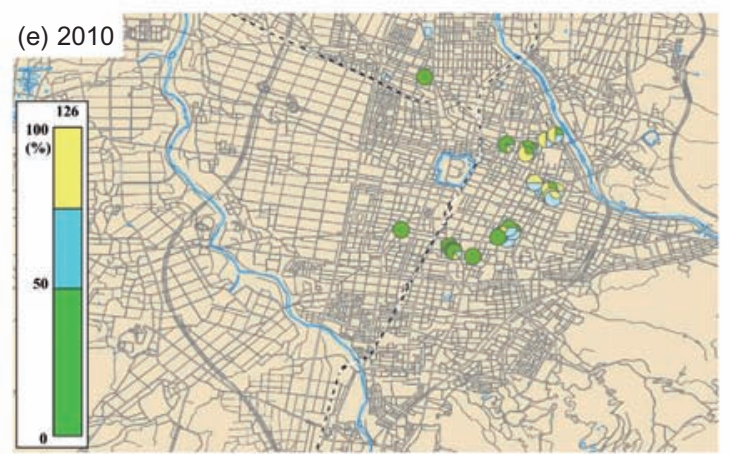

Fig. 4. Species proportions of collected mosquitoes in each collection site in 1998 (a), 2000 (b), 2002 (c), 2009 (d) and 2010 (e). There were three types of collection sites: Ae.j. japonicus only, Ae. albopictus only, and $\bullet$ Ae. albopictus + Ae. j. japonicus). The miniature pie chart circles indicate the position and the type of mosquito found at each collection site, each of which had many sampling points. The bar graphs show the species proportions collected during each survey with the number on top the total number of samples collected.

were collected from 126 colonies in 33 sites. Out of these, 60 colonies $(47.6 \%)$ consisted of only Ae. albopictus, 33 colonies $(26.2 \%)$ of only Ae.j.japonicus, and 33 colonies $(26.2 \%)$ of both species in coexistence (Fig. 4e). The results of the 2010 survey were almost the same as in 2009. Compared to the 2009 survey, the number of colonies with only Ae.j.japonicus mosquitoes did not change, while the number of exclusive Ae. albopictus colonies actually decreased. However, colonies with coexisting species increased. As a result, the habitats of Ae.j. japonicus colonies expanded.

\section{Environment confirmation using satellite images}

Changes in the environment in the 1990s and around 2009 were studied by comparing the JERS-1 and ALOS images. Multivariate correlation analyses compared data from the 1 October 1997 JERS-1 image and 20 September 2009 ALOS image (Table 2). Comparison of the NDVI values and patterns, which were obtained in practically corresponding months, revealed no significant changes in the environment, such as land use or urbanisation, between the 1990s and 2009.

NDVI diagrams were also created from ALOS images from 20 September 2009 using image quality and variables, such as cloud cover, state of paddy cultivation (the main form of land use), vegetation activity ( Fig. 5a). Division and colour coding of the NDVI into sectors revealed the distribution of areas with a NDVI $>0.08$ in the forests in the mountainous areas to the east of the survey area and in the paddy fields to the west. In the central urbanised areas, where areas with a NDVI $<-0.10$ prevail, a red square tracing the ruins of a castle can be seen. The results of the speci- 
Table 2. Correlations of the NDVI values of research areas studies by comparison of JERS-1 (J) and ALOS (A) images between 1993 and 2009.

\begin{tabular}{lcccccccccc}
\hline & A-080502 & A-080917 & A-081016 & A-090418 & A-090920 & J-931010 & J-950618 & J-951028 & J-971001 & J-980326 \\
\cline { 2 - 10 } A-080502 & - & - & - & - & - & - & - & - & - & - \\
A-080917 & 0.967 & - & - & - & - & - & - & - & - & - \\
A-081016 & 0.919 & 0.963 & - & - & - & - & - & - & - & - \\
A-090418 & 0.839 & 0.846 & 0.861 & - & - & - & - & - & - & - \\
A-090920 & 0.926 & 0.961 & 0.956 & 0.841 & - & - & - & - & - & - \\
J-931010 & 0.758 & 0.811 & 0.820 & 0.817 & 0.826 & - & - & - & - & - \\
J-950618 & 0.786 & 0.763 & 0.755 & 0.739 & 0.764 & 0.805 & - & - & - & - \\
J-951028 & 0.559 & 0.626 & 0.690 & 0.684 & 0.686 & 0.886 & 0.717 & - & - & - \\
J-971001 & 0.802 & 0.790 & 0.772 & 0.727 & 0.780 & 0.824 & 0.864 & 0.755 & - & - \\
J-980326 & 0.627 & 0.650 & 0.643 & 0.663 & 0.674 & 0.723 & 0.757 & 0.756 & 0.826 & - \\
\hline
\end{tabular}

men collection survey show that areas of Ae. albopictus habitation are numerous where the NDVI values are -0.06 to 0.01 or in the areas with a NDVI $>0.01$ that are dotted around the urbanised area (Fig. 5b).

The habitat areas, calculated from a 1:2,500-scale map using ArcView, varied considerably (258 to $6,667 \mathrm{~m}^{2}$ ) between the different sites sampled around an average of $1,913 \mathrm{~m}^{2}$ (Table 3 ). Over the entire survey area, the average area of the 12 sites where Ae.j.japonicus and Ae. albopictus coexisted was confirmed at $1,849 \mathrm{~m}^{2}$. The habitat area where only Ae. albopictus was found was $894 \mathrm{~m}^{2}$, while the area where only Ae.j.japonicus was found was $3,530 \mathrm{~m}^{2}$. Although only a small number of sites were surveyed, there was still a territorial difference between the species. The area derived from data used to calculate the NDVI values was $300-6,500 \mathrm{~m}^{2}$, indicating that an approximation of area can easily be compiled from values calculated from the NDVI.
When the NDVI values were shown as averages for each habitat using the 20 September 2009 images, the average NDVI value was -0.053 (standard deviation $(\mathrm{SD})=0.071 ;$ range $=-0.153-0.115)$. The average NDVI for the survey areas where Ae. albopictus habitation was confirmed was $-0.065(\mathrm{SD}=0.057$; range $=$ -0.153-0.064) and the average NDVI value for survey areas where Ae.j. japonicus was found was -0.050 (SD $=0.078$; range $=-0.153$ to 0.115$)$ indicating a slight trend for higher NDVI values in Ae.j. japonicus compared with Ae. albopictus habitat (Table 4). However, there was no significant difference by Turkey-Kramer's HSD test for 2010. Looking at the NDVI results for the range of habitation polygons estimated from the 2009 Ae. albopictus and Ae.j.japonicus collection sites and the $100 \mathrm{~m}$ radius circles (Table 5), the average respective mean NDVI values were $-0.053(\mathrm{SD}=0.071)$ and $-0.104(0.047)$, the average maximum values were 0.006 and 0.084 , the average minimum values were
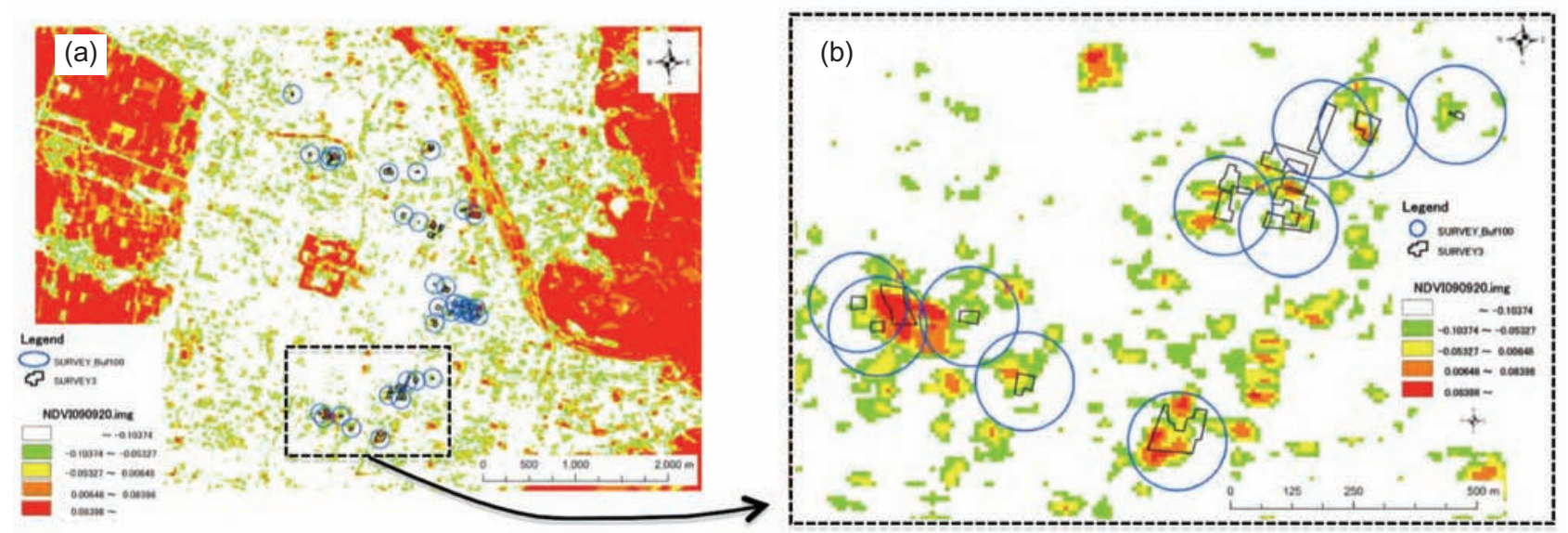

Fig. 5. NDVI diagram created from ALOS image of 19 September 2009. NDVI values were classified into five ranges based on the statistic analysis of the NDVI values of Aedes habitats. The polygons show the estimated infested areas and the circles the $100 \mathrm{~m}$ buffer zones around the collection sites. Overview including all collection sites (a) with a blow-up of the are in the rectangle (b). 
Table 3. Mosquito species collected in 2009 and estimated infested area.

\begin{tabular}{|c|c|c|c|}
\hline \multirow{2}{*}{ Sampling site } & \multirow{2}{*}{ Mosquito species } & \multicolumn{2}{|c|}{ Area $\left(\mathrm{m}^{2}\right)$} \\
\hline & & GIS* & $\mathrm{RS}^{* *}$ \\
\hline Yakushidou & Ae.j.japonicus & $6,667.44$ & $6,500.0$ \\
\hline Ryumonji & Ae.j.japonicus & $3,474.33$ & $3,400.0$ \\
\hline Taizoji & Ae.j.japonicus & 447.59 & 800.0 \\
\hline Mean & & 3529.75 & $3,566.7$ \\
\hline Hakuzanji & Ae.j. japonicus + Ae. albopictus $+* * *$ & $1,303.02$ & $1,300.0$ \\
\hline Ennoji & Ae.j. japonicus + Ae. albopictus $+* * *$ & $2,938.45$ & $2,900.0$ \\
\hline Jourinji & Ae.j.japonicus + Ae. albopictus +*** & $2,307.38$ & $2,400.0$ \\
\hline Bonkoji & Ae. j. japonicus + Ae. albopictus $+* * *$ & $4,629.66$ & $4,300.0$ \\
\hline Houonji & Ae.j. japonicus + Ae. albopictus $+* * *$ & 512.01 & 600.0 \\
\hline Genmyoji & Ae. j. japonicus + Ae. albopictus $+* * *$ & 733.87 & 600.0 \\
\hline Jyogenji & Ae. j. japonicus + Ae. albopictus $+* * *$ & 944.50 & $1,000.0$ \\
\hline Entokuji & Ae. j. japonicus + Ae. albopictus $+* * *$ & 258.18 & 300.0 \\
\hline Jyouzenji & Ae.j.japonicus + Ae. albopictus $+* * *$ & $1,124.61$ & $1,100.0$ \\
\hline Mean & & $1,639.08$ & $1,611.0$ \\
\hline Jikoji & Ae.j.japonicus + Ae. albopictus & $3,679.44$ & $3,600.0$ \\
\hline Joukoji & Ae.j.japonicus + Ae. albopictus & $1,325.15$ & $1,300.0$ \\
\hline Senshouji & Ae.j.japonicus + Ae. albopictus & $2,430.54$ & $2,400.0$ \\
\hline Mean & & 2478.38 & 2433.3 \\
\hline Shoukakuji & Ae. albopictus & $1,161.00$ & $1,200.0$ \\
\hline Shouinji & Ae. albopictus & $1,241.45$ & $1,200.0$ \\
\hline Denshoji & Ae. albopictus & 705.91 & 800.0 \\
\hline Enmeiji & Ae. albopictus & 467.35 & 500.0 \\
\hline Mean & & 893.93 & $1,233.3$ \\
\hline Total & & $1,913.26$ & $1,905.3$ \\
\hline
\end{tabular}

*Geographical information systems; **remote sensing; ***mixed species in same colony.

-0.113 and -0.195 , and the average variance was 0.120 and 0.279 . Looking at the correlations between these values for the polygons and circles, the mean value was 0.860 , the maximum value was 0.784 , the minimum value was 0.229 and the variance was 0.613 .

Here, as the correlation coefficients for the mean and maximum values were high, these latter values were used to classify NDVI and to create a map. September NDVI values for the Ae.albopictus and Ae.j.japonicus habitats were set at the following threshold values: maximum, 0.084 and 0.006 , respec- tively; mean, -0.053 and -0.104 , respectively (Fig. 5a). While the map included all high NDVI value areas within the polygons and the $100 \mathrm{~m}$ radius circles, the minimum value of -0.195 corresponded to an area that is extensively urbanised (Fig. 5b), which included a range where, empirically, no mosquito larvae were collected. Although the area with NDVI values ranging between -0.104 and -0.053 is urbanised, it is accepted that mosquitoes are present; hence, the distribution map was created on the premise that any area with a high NDVI constituted a habitat.

Table 4. NDVI values in the polygon covering the estimated infested area.

\begin{tabular}{llcccc}
\hline No. of sites & Species & Mean DVI & STD & Max & Min \\
\hline 15 & Ae. j. japonicus & -0.050 & 0.078 & 0.115 & -0.153 \\
12 & Mixed species* & -0.065 & 0.063 & 0.064 & -0.153 \\
16 & Ae. albopictus & -0.065 & 0.057 & 0.064 & -0.153 \\
3 & Ae. j. japonicus only & 0.009 & 0.121 & 0.115 & -0.122 \\
4 & Ae. albopictus only & -0.065 & 0.039 & -0.031 & -0.122 \\
& Total & -0.053 & 0.071 & 0.115 & -0.153 \\
\hline
\end{tabular}

\footnotetext{
"In same colony
} 
Table 5. Correlation of NDVI values between the polygon covering the estimated infested area and the $100 \mathrm{~m}$ buffer zone.

\begin{tabular}{lccccc}
\hline & Mean & SD* & Max & Min & Range \\
\cline { 2 - 6 } Polygon & -0.053 & 0.071 & 0.006 & -0.113 & 0.120 \\
Buffer zone & -0.104 & 0.047 & 0.084 & -0.195 & 0.279 \\
Correlation coefficient & 0.860 & - & 0.784 & 0.229 & 0.613 \\
\hline
\end{tabular}

*Standard deviation

\section{Discussion}

Although the Yamagata city's latitude is low for a northern mosquito limit region, the usual pattern of northward Ae.albopictus habitat migration due to global warming does not apply due to the particular geographical situation there. Yamagata city is a "heat island" located in an isolated basin surrounded by mountain with generally low temperatures; indeed too low for Ae. albopictus habitats so the mosquito cannot migrate unaided here from other habitats. However, this region now meets its minimum habitat temperature condition of $11^{\circ} \mathrm{C}$. Even though the temperature is now suitable for Ae. albopictus habitation, its presence was not confirmed at the start of our study. Over a period of more than 10 years, we repeatedly studied mosquito population dynamics, focusing on Ae. albopictus infestation and subsequent establishment status and interspecies competition between Ae.albopictus and Ae.j.japonicus, the indigenous priority mosquito species in the area.

Although Ae. albopictus infested and became established in regions inhabited by the indigenous species Ae.j.japonicus, these are clearly threshold temperature areas, and when a cold summer typical of the Tohoku region occurs, conditions become disadvantageous for Ae. albopictus habitation. The indigenous species Ae.j.japonicus, which is adapted to low temperatures, has a wider distribution and a longer breeding season than Ae. albopictus. Analysis of the geographical position of the survey area indicates that it is isolated from regions where Ae.albopictus is established. Expansion of Ae. albopictus habitat, other than through unaided means, generally occurs along expressways and arterial roads. The survey area is an agricultural area, and there is no industry requiring frequent travel to and from regions where Ae. albopictus has already been established. In fact, traffic volume censuses and maps from 1999 and 2005 show that few vehicles travelled from regions where Ae. albopictus has been established to the survey area. For these reasons, the survey area had little influx from other populations after the initial Ae. albopictus infestation and the increase in habitation density has thus been restricted. Although Ae. albopictus was expected to take over, Ae.j.japonicus once again expanded its habitat, and further surveys conducted 2009-2010 reveal that larvae from both species frequently coexisting in the same water body.

Although interspecies competition is presumed to depend upon habitation density with regard to the larval stages, there is no indication at present that one species is eliminating the other. However, if temperatures continue to increase resulting in longer breeding seasons and an increased number of Ae.albopictus generations per year, its habitation density will most probably increase thereby accelerating this mosquito population.

In recent years, surveys of mosquito establishment status have been conducted in North America and Europe, where both Ae. albopictus and Ae.j. japonicus occur (Joy and Sullivan, 2005; Juliano and Lounibos, 2005; Grim et al., 2007; Farajollahi and Nelder, 2009; Kesavaraju et al., 2011). For example, temperatures were not discussed in detail, the results of a survey using ovitraps near Weil am Rhein in Germany near the border with Switzerland $\left(47^{\circ} 42^{\prime}\right.$ $22 " \mathrm{~N}$ ), showed that Ae.j. japonicus has been established, and although Ae. albopictus eggs were found, adults were not, indicating that Ae.albopictus was not established (Becker et al. 2001). However, according to a survey undertaken by the authors, the annual mean temperature in this place is $9{ }^{\circ} \mathrm{C}$, which is below the minimum temperature suitable for Ae. albopictus habitation. These conditions are considered similar to those in Yamagata city before 1945, and we would like to maintain a focus on the relationship between increasing temperatures and mosquito establishment.

In this study, Ae. albopictus larva surveys were conducted in relation to artificial vessels and other areas where water can accumulate, located in urban areas where temples and surrounding trees provide partial shading. As this is an urban area, which was not surveyed as land use and building types render mosquito habitation there impossible, the GIS method of divid- 
ing the area into a mesh pattern and searching for a definite number of samples and then collecting larvae within a mesh grid was not applicable. We therefore estimated green spaces adjacent to larva collection sites by deciphering aerial photographs and basic national land use maps to set the habitation range. However, as this method was complicated, we further developed a simple method that anyone can apply, i.e. we drew circles with a $100 \mathrm{~m}$ radius centring on the centroids of polygons, set the NDVI values derived from these as the threshold values and determined which was more valid for estimating habitat.

As we will see if we compared with the polygon to the $100 \mathrm{~m}$ radius circle in Fig. 5, the $100 \mathrm{~m}$ radius circles contained habitat areas as well as urbanised areas not useful for mosquito survival. In particular, as there was a maximum 100- to 300 -fold difference in the sizes of these areas, there were also significant variations in minimum NDVI values between the two. The minimum values were thus thought to include false negatives in terms of the estimated habitation range and false positives for the circles. Consequently, we used average and maximum values for this period for which correlations were confirmed. In particular, areas with maximum values are thought to always include places where mosquitoes can find suitable environments.

The JERS- 1 and ALOS satellites launched by Japan have higher observation frequencies than maps and aerial photographs, higher image resolution, and also cost less in comparison to satellite images from other countries, which make them more convenient to use. In addition, satellite images allow the deciphering of NDVI values, which cannot be captured using maps or aerial photographs.

Satellite images are useful for the analysis of environmental changes in areas in different eras. However, with regard to image criteria, since the wavelengths that can be obtained differ depending on satellite type, these images are not always suited to comparisons across different time periods. For the current study, we gathered and compared as many images relating to the survey area as we could obtain from the JERS-1 satellite and from the subsequent ALOS satellite launched from Japan, selecting images that corresponded to sample collection seasons. We believed that the estimated habitat range of Ae. albopictus could be estimated from the NDVI values calculated from satellite images corresponding to the sample collection periods. Using these images, we ascertained that there were no significant environmental changes since 1998, and inferred that Ae.albopictus habitat was expanding because of changes in temperature due to global warming.

Although the habitats of both species were regions with comparatively high NDVI values, since the number of colonies where the two species lived separately were few, classification of the habitat of the two species according to NDVI values was not significant. However, we did ascertain that Ae.j. japonicus tended to inhabit wide-ranging areas with high NDVI values, and that Ae. albopictus tended to inhabit smaller areas with lower NDVI values. We were able to confirm differences in geographical distribution, with the Ae. j. japonicus population occurring high in the green areas near the primary habitat of Ae.j. japonicus to the east of the basin at the foot of the mountains, while the Ae. albopictus population was in high areas further away from these areas, many near Japan railway stations and government and public offices, in lower NDVI value areas where urbanisation projects in recent years have extended roads and where urban areas have expanded.

\section{Conclusions}

Within marginal habitat areas in terms of temperature conditions, we surveyed the progress of Ae. albopictus infestation attributable to the formation of heat islands resulting from global warming, through field surveys and GIS/remote sensing. In the initial stages of Ae. albopictus infestation, we believed that the indigenous species, Ae. j. japonicus, would be driven out. However, even with global warming, there were still annual fluctuations in temperature and there were some years, particularly those with cold summers, when Ae. albopictus development was restricted as the effective accumulative temperature fell below the $11{ }^{\circ} \mathrm{C}$ threshold for Ae. albopictus. Consequently, Ae.j.japonicus, which has a greater tolerance to lower temperatures, returned to the initially expanding Ae. albopictus habitat, and within one of the survey areas, we observed colonies of both species coexisting as well as one colony in which both species co-existed. The competitive relationship between these two Aedes species within a warming environment is an issue that should be closely observed in the future. Meanwhile, we were successful in using GIS to decipher and map Aedes habitat. Henceforth, we will be looking to implement outdoor surveys while checking the habitat of both Aedes species using this map, in order to verify the status of competition between the two species in their outdoor habitat instead of under laboratory conditions. 


\section{Acknowledgements}

This work was partially supported by a grant from "Global Environmental Research Fund (S-8)" by the Ministry of Environment Japan and from the Ministry of Health, Labor and Welfare of Japan (H18-Shinko-Ippan-009).

\section{References}

Armistead JS, Arias JR, Nishimura N, Lounibos LP, 2008. Interspecific larval competition between Aedes albopictus and Aedes japonicus (Diptera: Culicidae) in northern Virginia. J Med Entomol 45, 629-637.

Becker N, Huber K, Plusckota B, Kaiser A, 2011. Ochlerotatus japonicus japonicus - a newly established neozoan in Germany and a revised list of German mosquito fauna. European Mosq Bull 29, 88-102.

Benedict MQ, Levine RS, Hawley WA, Lounibos LP, 2007. Spread of the tiger: global risk of invasion by the mosquito Aedes albopictus. Vector Borne Zoonotic Dis 7, 76-85.

Burt FJ, Rolph MS, Rulli NE, Mahalingam S, Heise MT, 2012. Chikungunya: a re-emerging virus. Lancet 379, 662-671.

ECDC, 2009. Development of Aedes albopictus risk maps. Stockholm: ECDC Technical Report, 45 pp.

Farajollahi A, Nelder M, 2009. Changes in Aedes albopictus (Diptera: Culicidae) populations in New Jersey and implications for arbovirus transmission. J Med Entomol 46, 1220-1224.

Gratz N, 2004. Critical review of the vector status of Aedes albopictus. Med Vet Entomol 18, 215-227.

Grim DC, Jackson BT, Paulson SL, 2007. Abundance and bionomics of Ochlerotatus j. japonicus in two counties in southwestern Virginia. J Am Mosq Control Assoc 23, 259-263.

Hawley WA, 1989. The biology of Aedes albopictus. J Am Mosq Control Assoc 4, 1-40.

Helinski MEH, Deewatthanawong P, Sirot LK, Wolfner MF, Harrington LC, 2012. Duration and dose-dependency of female sexual receptivity responses to seminal fluid proteins in Aedes albopictus and Ae. aegypti mosquitoes. J Insect Physiol 58, 1307-1313.

Japan Meteorological Agency, 1993. Climate table of Japan, vol. 2. 10 days/5 days/daily averaged values in an average year. Tokyo, Japan (in Japanese).

Joy JE, Sullivan SN, 2005. Occurrence of tire inhabiting mosquito larvae in different geographic regions of West Virginia. J Am Mosq Control Assoc 21, 380-386.

Juliano SA, Lounibos LP, 2005. Ecology of invasive mosquitoes: effects on resident species and on human health. Ecol Lett 8 , 558-574.

Kesavaraju B, Khan DF, Gaugler R, 2011. Behavioral differ- ences of invasive container-dwelling mosquitoes to a native predator. J Med Entomol 48, 526-532.

Kobayashi M, Komagata O, Nihei N, 2008. Global warming and vector-borne infectious diseases. J Disaster Res 2, 105112.

Kobayashi M, Nihei N, Kurihara,T, 2002. Analysis of northern distribution of Aedes albopictus (Diptera: Culicidae) in Japan by geographical information system. J Med Entomol 39, 4-11. Kurihara T, Kobayashi M, Kosone T, 1997. The northward expansion of Aedes albopictus distribution in Japan. Med Entomol Zool 48, 73-77.

Mori A, 1979. Effects of larval density and nutrition on some attributes of immature and adult Aedes albopictus. Trop Med 21, 85-103.

Paupy C, Girod R, Salvan M, Rodhain R, Failloux AB, 2001. Population structure of Aedes albopictus from La Reunion Island (Indian Ocean) with respect to susceptibility to a dengue virus. Heredity 87, 273-283.

Raineri V, Trovato GT, Sabsatini A, Coluzzi M, 1991. Recent data on the infestation of Genoa with Aedes albopictus. Parassitologia 33, 183-185 (in Italian).

Renault P, Solet JL, Sissoko D, Balleydier E, Larrieu S, Filleul L, Lassalle C, Thiria J, Rachou E, de Valk H et al., 2007. A major epidemic of chikungunya virus infection on Reunion Island, France, 2005-2006. Am J Trop Med Hyg 77, 727-731.

Sato T, Matsumoto F, Abe T, Nihei N, Kobayashi M, 2012. Recent distribution of Aedes albopictus in Iwate Prefecture, northern Japan and analysis of climatic conditions for northern limit of geographic distribution by geographic information systems. Jpn J Sanit Zool 66, 195-204 (in Japanese).

Schaffner F, Chouin S, Gutlloteau J, 2003. First record of Ochrerotatus (Finlaya) japonicus japonicus (Theobald, 1901) in metropolitan France. J Am Mosq Control Assoc 19, 1-5.

Tohoku Agricultural Research Center, NARO, 2009. 1-km mesh temperature data display/retrieval system for Tohoku Region. Available at: http://www.naro.affrc.go.jp/ org/tarc/seika/jyouhou/H13/tohoku01028.html (accessed on February 2005) (in Japanese).

Tripet F, Lounibos P, Robbins D, Moran J, Nishimura, N, Blosser EM, 2011. Competitive reduction by satyrization? Evidence for interspecific mating in nature and asymmetric reproductive competition between invasive mosquito vectors. Am J Trop Med Hyg 85, 265-270.

Udaka M, 1959. Some ecological notes on Aedes albopictus Skuse in Shikoku, Japan. Kontyu 27, 202-208 (in Japanese).

Werner D, Kronefeld M, Schaffner F, Kampen H, 2012. Two invasive mosquito species Aedes albopictus and Aedes japonicus japonicus, trapped in south-west Germany, July to August 2011. Euro Surveill 17, 4. 